\title{
Enhancement of a Body Area Network to support Smart Health monitoring at the digital home
}

\author{
Vadillo, Laura \\ $\mathrm{T}>\mathrm{SIC}$ \\ Universidad Politécnica de Madrid \\ Ctra. Valencia, Km.7 \\ 28031, Madrid, Spain \\ +34 913365526 \\ Ivadillo@diatel.upm.es
}

\author{
Valero, Miguel A. \\ DIATEL \\ Universidad Politécnica de Madrid \\ Ctra. Valencia, Km.7 \\ 28031, Madrid, Spain \\ +34913367820 \\ mavalero@diatel.upm.es
}

\author{
Gil, Gema \\ Primary Care Service of \\ Perales de Tajuña \\ C/ Mayor, 13. Valdilecha \\ 28511, Madrid, Spain \\ +34 918738579 \\ ggiljuberias@gmail.com
}

\begin{abstract}
The deployment of home-based smart health services requires effective and reliable systems for personal and environmental data management. Cooperation between Home Area Networks (HAN) and Body Area Networks (BAN) can provide smart systems with ad hoc reasoning information to support health care. This paper details the implementation of an architecture that integrates BAN, HAN and intelligent agents to manage physiological and environmental data to proactively detect risk situations at the digital home. The system monitors dynamic situations and timely adjusts its behavior to detect user risks concerning to health. Thus, this work provides a reasoning framework to infer appropriate solutions in cases of health risk episodes. Proposed smart health monitoring approach integrates complex reasoning according to home environment, user profile and physiological parameters defined by a scalable ontology. As a result, health care demands can be detected to activate adequate internal mechanisms and report public health services for requested actions.
\end{abstract}

\section{Categories and Subject Descriptors}

C.2.4 [Distributed Systems]: Distributed applications; F.4.3 [Formal Languages]: Decision problems; H.4.2 [Types of Systems]: Decision support; I.2.11 [Distributed Artificial Intelligence]: Intelligent agents - Multiagent systems.

\section{Keywords}

Smart Health, context aware system, multiagent home platform

\section{INTRODUCTION}

Enhancements in life expectancy could benefit from emerging Information and Communication Technologies at home to support health care in public health scenarios. World Health Organization points out that the number of ageing people will be double in 2025 , which represents about $22 \%$ of the world population in 2050. This demographic change influences the prevalence of chronic conditions and diverse diseases. Aging population demands qualified care and personnel to efficiently face health needs like continuous attention that requires sustainable health care models.

Permission to make digital or hard copies of all or part of this work for personal or classroom use is granted without fee provided that copies are not made or distributed for profit or commercial advantage and that copies bear this notice and the full citation on the first page. To copy otherwise, to republish, to post on servers or to redistribute to lists, requires prior specific permission and/or a fee. BODYNETS 2013, September 30-October 02, Boston, United States Copyright @ 2013 ICST 978-1-936968-89-3

DOI 10.4108/icst.bodynets.2013.253578
The provision of high quality, cost effective, specialized and accessible systems for the population are still challenges to be faced by global healthcare systems. In this sense, the reliable interaction between smart-home technologies and biomedical devices becomes an essential aspect to enhance user's quality of life for people with critical health care needs [1] [2] [3].

Health monitoring systems gather patient's status to provide health professionals in a remote center with medical data. Many research aimed to acquire remote vital signals by using BAN to monitor health status in telehealth, rehabilitation, sports or training projects [4]. Jung et al showed in 2008 how the detection of risk situations at a home telehealth scenario can be significantly improved by including data collected from the environment (situation of the person in the house, $\mathrm{CO} 2$ detection, etc.) [5]. Aforementioned system employed environmental sensors and vital signs devices to manage patient's health status. However, it is not enough to gather information about users and their environment, by using BANs or other technologies. This information must be processed by context aware mechanisms that should react at any change and deduce dangerous states for the user, triggering necessary mechanisms that permit solving conflictive situations.

Common wearable devices are not able to carry out sophisticated reasoning by themselves. They need to be enhanced by ad hoc mechanisms to aggregate captured data and make reasoning. Multiagent systems are valuable for dynamic and inaccurate environments where the response time, efficiency and precision in data management is a key issue [6] [7]. Telehealth scenarios, like the one faced in the research detailed in this paper, fit to these needs and are still unsolved. Published solutions mainly aim at solving specific healthcare problems of concrete user profiles. On the other hand, decision-support-systems usually focus on biomedical data without taking into account the information of the environment. The system described in this paper allows the integration of wearable devices that monitor physical parameters, with environment sensors in order to detect health care risk situations in an effective way. Reasoning actions are based on ontologies created from use cases. Multiagent platforms frequently use ontologies to provide a semantic infrastructure for the communication between agents and to support the Knowledge Base (KB) in context aware systems. The use of ontologies as a tool for context definition have been widely validated in many systems such as the CoBra architecture [8] where a representation of the context information can be shared and processed thanks to the use of Web Semantic Languages (RDF and OWL). Recent health monitoring systems use ontologies to support home-based continuous care [9], although they do not integrate BANs like the work validated in this research. 


\section{MATERIALS AND METHODS}

The construction of a reasoning platform for Smart Health monitoring requires a context aware model to define the $\mathrm{KB}$ and the multiagent architecture. A development methodology based in CommonKADS [10] and MAS-CommonKADS was followed to create the platform. The first stage is the knowledge acquisition phase whose goal is to extract relevant information about health monitoring at home to build the KB. This work was done by creating forms according to the recommendations of the World Health Organization (WHO), the Spanish Institute for Elderly and Social Services (IMSERSO), the Andalusian Society of Geriatrics and Gerontology and the Spanish Red Cross. These forms were fulfilled by social workers and people who use home telecare services. Thus, working system variables, users' opinions and profiles of telecare users were collected to create the system KB.

The knowledge definition, its representation and the association of concepts needs the definition of reasoning mechanisms according to the acquired information. Three key aspects are involved in health monitoring: input information to be analyzed, information monitoring procedures, and expected system's outputs or tasks to be performed. Input information consists of the following user data sets: user profile that indicates his health status; physiological parameters; health indicators; and the detectable parameters at the home that can influence the health state of the person (fall, inactivity, smoke, gas, environment temperature). It is also necessary to include deductions and actions associated to the captured parameters to be managed by the reasoning platform. Therefore, it is necessary to exactly define the behavior of the digital home and how decisions should be taken when risk situations arise.

\section{SYSTEM RESULTS}

The architecture of the developed system, called TALISMAN+, is divided into three layers, differentiated through actions managed along the monitoring sequence. Sensors monitor context data and feed the Context Adapter Layer (CAL) with acquired information. The CAL gathers sensors data to be sent to the Reasoning Layer (RL), after being filtered and translated. The RL processes the message, which contains the information context, received by the CAL. This system incorporates a behavior model that manages the situation awareness model and shares the information with a group of agents that make inferences with the actual context in a reliable way. The reasoning agents process the incoming information collected by the sensors with the KB. Then, they can deduce new knowledge and add it to their KB, through their inferences engine. Inferences engine, integrated with Pellet in this research, can infer the degree of severity of the actual health context and make decisions, like to warn the urgency medical services if a complex combination of events is detected.

The architecture implementation (Figure 1) is built with a multiagent platform developed in JADE and installed in a laptop in the user's house. The system is composed by software cooperative entities, organized in different levels according to its functionality. Its capacity for parallel processing provides a reasoning performance that could not be achieved by a single agent in the same conditions. This structure can significantly increase the system performance as it improves the capacity of decision. Furthermore, urgent data prioritization and higher flexibility is obtained allowing the incorporation of new functionality in an easy way, including new agents or additional capabilities in the existing agents.

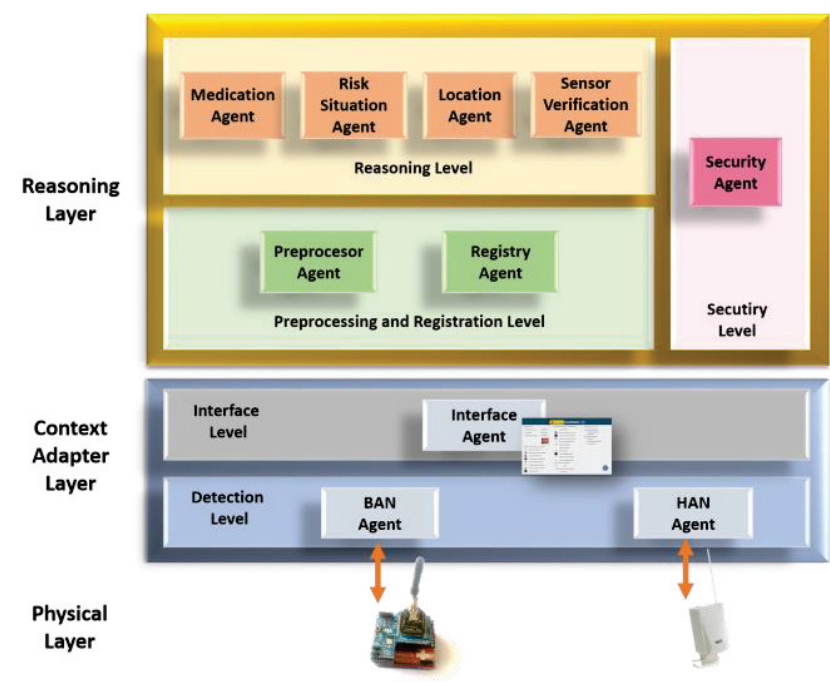

Figure 1. TALISMAN+ architecture.

\subsection{Components of the Physical Layer}

This layer is composed by physical devices sited in the house. This equipment can be divided in two main networks: Body Area Network (BAN) and Home Area Network (HAN). BAN sensors monitor person's physiological parameters such as glucose level, heart rate, body temperature, blood pressure and oxygen saturation, and send them to the BAN Agent, through a Bluetooth module connected with an Arduino eHealth Sensor Platform linked to an Arduino UNO board. HAN sensors gather measures of the user's environment such as his location in the house, bed or chair occupancy, fall of the user, carbon monoxide exposition, flood detection, temperature measurement or smoke detection.

The device that receives information of HAN sensors is CM15 Controller. CM15 controller can receive X10 or RF433 orders. This controller receives directly command of the X10 RF PIR Motion Sensors, and changes in X10 devices. The rest of the equipment are telecare devices running on the $869 \mathrm{MHz}$ radiofrequency channel. These devices send the information to a home unit. TALISMAN+ takes advantage of Tunstall home unit (Lifeline Connect+) since it provides an X10 interface that permits to map the states of the sensors with X10 orders. Through this orders, it is possible to know the change of a telecare sensor. Thus, it is necessary to include a gadget called My Life X10 adapter together with XM10 device, which acts as interface between the Lifeline Connect+ and the electrical line; and to program the home unit so it can send a different X10 command according to the activated sensor at each moment.

\subsection{Developed Context Adapter Layer}

This layer is the interface between the RL and the Physical Layer. CAL is composed by different agents depending on the communication needs with each device. In each case, the system incorporates three main agents at two different levels: Body Area Network Agent (BAN Agent) and Home Area Network Agent (HAN Agent) at the Detection Level, and the Interface Agent at the Interface Level. The BAN Agent collects information from the sensors incorporated in the Body Area Network explained in the previous subsection. The HAN Agent receives X10 orders, sends them by the telecare sensors through Lifeline Connect + or sends them through X10 devices. The Interface Agent placed at the 
Interface Level collects every change of the sensors sent by BAN Agent and HAN Agent, and shows the context state in a graphical user interface (GUI). In the same way, the Interface Agent collects the results of the inferences made in the Reasoning Module in form of action proposals, represents this action proposals in the GUI, and sends X10 commands to the actuators to turn off some solenoid (water or gas), or issue any warning or sound in the house. Figure 2 shows the three areas of the GUI: Personal profile of the user, Sensors included in the system, and Action Proposals triggered when a context change happens. Data acquired through the BAN feed the biomedical sensors area providing glucose, health rate and blood pressure state, among others.

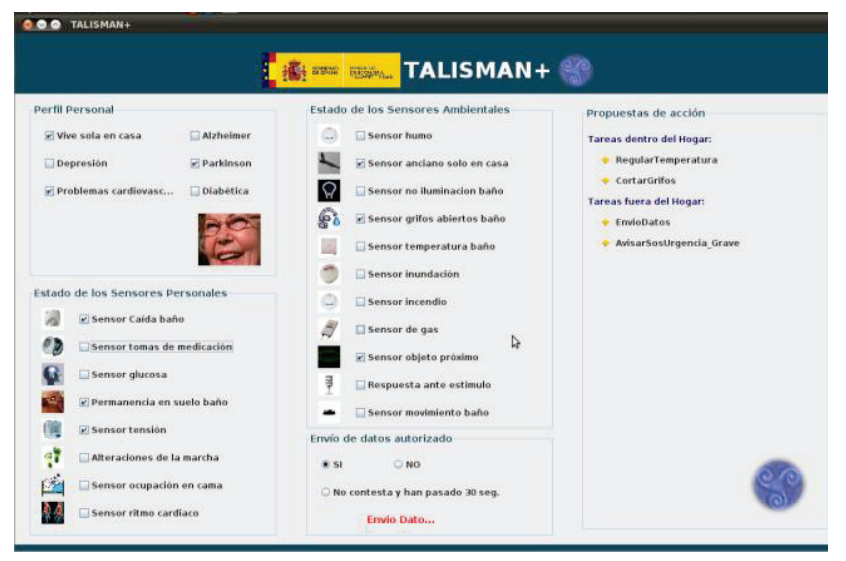

Figure 2. Graphical User Interface of TALISMAN+.

Moreover, the interface includes an option to authorize data sending out of the house. This facility allows the user to decide when he or she wants the data can be sent. Thereby, the user knows the data that his/her house interchanges with the exterior, according to ETSI standard and the Spanish law of Personal Data Protection (LOPD).

\subsection{Implemented Reasoning Layer}

The e-Care Reasoning Module is composed by three main levels: Preprocessing and Registration, Reasoning and Security.

The Preprocessing and Registration Level starts with the actuation of Preprocessor Agent. This agent receives the changing information of a sensor, and sends the useful information to the Reasoning Level. It includes each change in a XML document in which every information of the context is registered, with a processing algorithm. The message includes some attributes such as: agent identification, time stamp (that shows the moment of message creation, when the context is changed) and the information of each sensor (sensor identification, actual value, activated time and activation moment if the sensor is activated). This XML document is sent to the Registry Agent, which stores this document together with previous contexts, including it in a registry that records each changed context situation over time.

The Reasoning level analyzes data from the Preprocessor Agent and infers knowledge based on the sensor states. It is composed by four agents: Medication Agent, Location Agent, Sensor Verification Agent and Risk Situation Agent. Location Agent analyzes data from X10-RF PIR motion sensors, and bed/chair occupancy. This Agent determinates person location at home, and analyzes if the person is alone, accompanied or if inconsistent data appears coming from the sensors. The Medication Agent includes a timetable with medication and monitors the medication control dispenser. Sensor Verification Agent manages inconsistent situation, testing the sensors states. This last Agent receives advertisement of the sensor state such as low battery. Lastly, the Risk Situation Agent includes information of Preprocessor Agent and other Agents in the same level. This agent is the most sophisticate. It uses an ontology defined by OWL, which contains the definition of the risk situation and state of gravity in function of sensors states and person profile (diseases and treatment known). Thus, the inference cycle is completed. When a sensor state changes, the Risk Situation Agent receives the context status from other agents (Preprocessor, Location and Medication) and contrasts this situation with the risk situation definition included in the ontology.

The Health Monitoring Ontology (HMO) (Figure 3) defines each situation as a set of sensor states by detailing the tasks to be performed by the system according to the situation at each temporal instant. Thus, the system reasoning is defined by patterns, which can be easily identified by the reasoning engine. A classical system of conditions structure reduces the system performance in proportion to the number of conditionings. The reasoning needs of the module, in which it is necessary to process a high number of sensors with different values, and with high speed data processing in a risk situation, rejects its use.

The use of ontologies makes possible to include new sensors, new profiles and new procedures for easy and efficient reasoning. Concepts hierarchy of the HMO is implemented through four main classes: SystemReport, covering the system states (quiet, risk, alarm, and the three possible alarm cases classified according dangerous); Virtual Sensor, that is an abstraction of any combination of physical sensors that will indicate a certain system state (active or not active); Personal Profile, that define the personal situation, life conditions and health problems and diseases of the user, which can have repercussions in the kind of virtual sensor considered; Security Wrapper, that define the security framework user-center; and Problem Solving Task, which are solutions chosen by the system depending on the criteria and sensors states (to notify the emergency services, to close taps, warning the person, remembering medication, etc. ). The ontology implementation was carried out with the support of the ontologies development environment called Protégé and the used Inference Engine is Pellet reasoner. To manage the ontology in Java-Jade, Jena and Protégé-owl API was used.

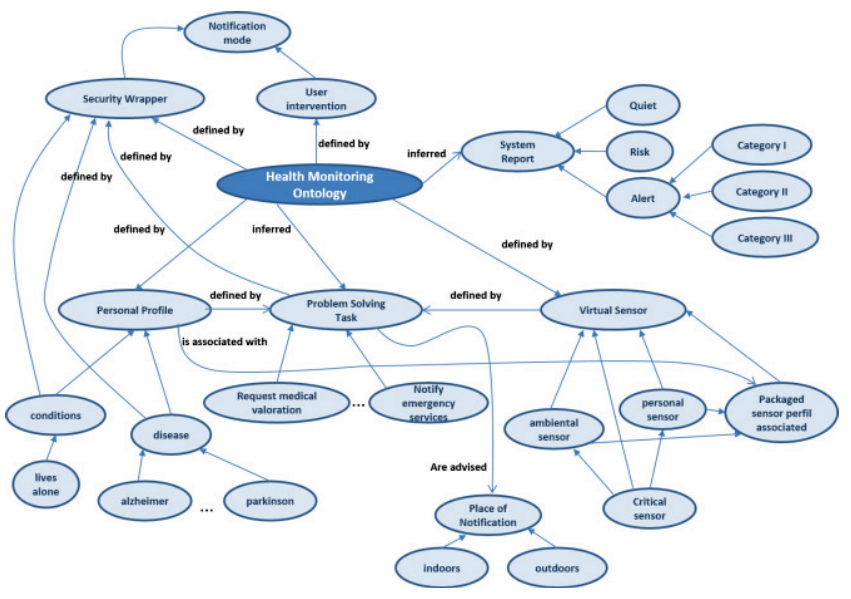

Figure 3. Health Monitoring Ontology. 
The mission of the Security Level is to guarantee the confidentiality of the information transmitted and stored in the system, the data integrity and authenticity both inside and outside of the home. On the one hand, it manages data securization in the transmitted messages between agents, and in the data to be stored in the system. And, on the other hand, the Security Agent sends cyphered information in external communications and includes a registry of conversation held between the house and caregiver. This registry allows to knowing all data exchanged. Thus, if the communication is closed by other causes, it is possible to resume it from the instant it was closed. Each external communication is registered in a XML document, which includes a timestamp and two types of transactions (messages), send and reception, a field of ask or response, and other field with the information.

\subsection{System Validation}

The implemented HMO was validated according to the test procedure proposed by Rational Unified Process. TALISMAN+ was connected with the HAN of the Accessible Digital Home at Universidad Politécnica de Madrid. Use cases were verified starting from the $2^{26}$ combinations of input variables that come from the HAN and BAN, which define each context situation. TALISMAN+ outputs were validated by comparing the proposed decision with the expected behavior that was previously specified in the knowledge acquisition phase. Processing time and actions proposed by the reasoner, according to the sensors states, were measured at the validation stage. The achieved delay between a physical sensor change and the activation of an action proposal in TALISMAN+ interface is always below 3 seconds. The inference time varies from 0.010 to 1.09 seconds. The highest inference time is the time requested by the system to start by itself once it has been accidentally closed. Current validation trials show the existence of some cases in which the obtained information is not accurate enough to understand the situation at the house.

\section{CONCLUSIONS}

The proposed TALISMAN+ architecture shows how BANs can be enhanced by smart monitoring systems. A home based context aware model explains and manages the system knowledge to support medical care. The knowledge acquisition phase, based on health experts and telecare service users, was a key aspect to build the KB. This phase allowed to link actions and states according to the available data provided by BAN and HAN subsystems.

The implemented three levels architecture, divided in agents according to functionality, provides a flexible and efficient data processing facility. TALISMAN+ instantaneously processes both environment and physiological data of the user collected through market available devices. Thus, it allows an early detection of risk situations and its notification to external health professionals.

The reaction time of the system, due to a sensor's state change or the necessity to be restarted, is highly satisfactory for the stated health care needs. The largest delay of three seconds is caused between the Physical Layer and Context Adapter Layer. As to the system accuracy, the ratio of cases in which the system proposes an erroneous gravity degree is low but needs to be measured and checked in more detail. Current research works aim to solve these cases by testing other technics, such as fuzzy logic, to obtain appropriate deductions at not accurate contexts.

\section{ACKNOWLEDGMENTS}

Authors would like to thank National Plan for Science, Development and Innovation of the Spanish Ministry of Science and Innovation which funds TALISEC+ project (TIN2010-20510C04-01) whose outcomes were essential for this research.

\section{REFERENCES}

[1] Rashidi, P. and Mihailidis, A. 2013. A survey on AmbientAssisted Living Tools for Older Adults. IEEE J. of Biomedical and Health Informatics 17, 3 (May 2013), 579590. DOI= 10.1109/JBHI.2012.2234129

[2] Alwan, M., Dalal, S., Mack, D., Kell, S.W., Turner, B., Leachtenauer, J. and Felder, R. 2006. Impact of monitoring technology in Assisted Living: Outcome Pilot. IEEE Trans. Inform. Technol. Biomed. 10, 1 (Jan. 2006), 192-198. DOI= 10.1109/TITB.2005.855552

[3] Sadri, F. 2011. Ambient intelligence: A survey. $A C M$ Comput. Surv. 43, 4, Article 36 (October 2011), 66 pages. DOI $=10.1145 / 1978802.1978815$

[4] Chen, M., Gonzalez, S., Vasilakos, A., Cao, H. and Leung, V.C. 2011. Body Area Networks: A Survey. J. Mob. Netw. Appl. 16, 2 (April 2011), 171-193. DOI= http://dx.doi.org/10.1007/s11036-010-0260-8

[5] Jung, J., Ha, K., Lee, J., Kim, Y. and Kim, D. 2008. Wireless body area network in a ubiquitous healthcare system for physiological signal monitoring and health consulting. International Journal of Signal Processing, Image Processing and Pattern Recognition, 1, 1 (Dec. 2008) 47-54.

[6] Horling, B. and Lesser, V. 2004. A survey of multi-agent organizational paradigms. J. Knowl. Eng. Rev. 19, 4 (Dec. 2004), 281-316. DOI= http://dx.doi.org/10.1017/S0269888905000317

[7] Nelson, J. and Moreno, A. 2003. Agent-Based Applications in Health Care. In Applications of Software Agent Technology in the Health Care Domain. Witestein Series in Software Agent Technologies, Birkhäuser Basel, 3-18. DOI= http://dx.doi.org/10.1007/978-3-0348-7976-7_2

[8] Chen, H., Finin, T., Joshi, A. 2004. Semantic Web in the context broker architecture. In Proceedings of the Second IEEE Annual Conference on Pervasive Computing and Communications 2004 (Florida, USA, March 14-17, 2004) PerCom 2004, 277-286. DOI= 10.1109/PERCOM.2004.1276865

[9] Paganelli, F., Giuli, D. 2011. An Ontology-Based System for Context-Aware and Configurable Services to Support HomeBased Continuous Care. IEEE Trans. Inf. Technol. Biomed. 15, 2 (March 2011), 324-333. DOI= 10.1109/TITB.2010.2091649

[10] Blanco, A.P., Valero M.A., Pau, I. 2008. Metodología de adquisición de conocimiento para telecuidado en el hogar digital. Collaborative Electronic Communications and eCommerce Technology and Research Iberoamérica (Madrid, Spain, June 25-27, 2008). CollECTeR 2008. 